\title{
Magnetic and Thermodynamic Properties and Spin-Flop-Driven Magnetodielectric Response of the Antiferromagnetic $\mathrm{Pb}_{2} \mathrm{Fe}_{2} \mathrm{Ge}_{2} \mathrm{O}_{9}$ Single Crystals \\ A.I.Pankrats $^{1,2}$, D.A.Balaev ${ }^{1,2}$, A.L.Freydman ${ }^{\text {, }}$, S.E.Nikitin ${ }^{3,4}$, A.A.Krasikov ${ }^{1}$, A.D.Balaev ${ }^{1}$, S.I.Popkov ${ }^{1,2}$, and M.I.Kolkov ${ }^{1,2}$ \\ ${ }^{1}$ Kirensky Institute of Physics, Federal Research Center KSC SB RAS, Krasnoyarsk, 660036 Russia \\ ${ }^{2}$ SiberianFederalUniversity, Krasnoyarsk, 660041 Russia \\ ${ }^{3}$ Max Planck Institute for Chemical Physics of Solids, Nöthnitzer Strasse 40, Dresden, 01187 Germany \\ ${ }^{4}$ Institut für Festkörper und Materialphysik, Technische Universität Dresden, Dresden, D-01069 Germany
}

\begin{abstract}
Orthorhombic $\mathrm{Pb}_{2} \mathrm{Fe}_{2} \mathrm{Ge}_{2} \mathrm{O}_{9}$ antiferromagnetic single crystals have been synthesized by a modified pseudo-flux technique and their magnetic, thermodynamic, and magnetodielectric properties have been investigated. It has been found that, below the Neel temperature $(45.2 \mathrm{~K})$, iron moments are arranged in a canted antiferromagnetic structure with a weak ferromagnetic moment parallel to the $\boldsymbol{a}$ axis. According to the specific heat measurement data, the $T_{\mathrm{N}}$ value remains invariable in applied magnetic fields of up to $5 \mathrm{~T}$ within the experimental accuracy. The magnetic entropy in the investigated crystals attains $2 R \ln (2 S+1)$ right above $T_{\mathrm{N}}$, which is indicative of a purely magnetic nature of the transition. It has been shown that the weak ferromagnetic moment is induced by the interplay between the single-ion anisotropy and antisymmetric Dzyaloshinskii-Moriya exchange interaction, with the latter contribution being dominant. It has been established from the angular dependences of the magnetization in three orthorhombic planes that the symmetries of the magnetic and crystal structure are identical. The magnetodielectric properties of the $\mathrm{Pb}_{2} \mathrm{Fe}_{2} \mathrm{Ge}_{2} \mathrm{O}_{9}$ single crystals have been studied at different mutual orientations of the electric and magnetic fields. The most prominent anomalies have been observed in the vicinity of the spin-flop transition in a magnetic field applied along the $\boldsymbol{c}$ axis.
\end{abstract}

\section{Introduction}

The search for new multifunctional materials and study of their physical properties are among the priority directions of modern solid-state physics. In particular, materials with the interrelated charge and spin degrees of freedom evoke a keen interest both as a basis for a new generation of microelectronic devices and an experimental playground for studying unexplored physical effects induced by the interplay of the electrical, magnetic, and elastic properties of materials.

The magnetic materials in which $3 \mathrm{~d}$ magnetic ions are combined with the so-called stereochemical ions are considered to be promising candidates for examining the multiferroic properties. Bright examples of the stereochemical ions are $\mathrm{Bi}^{3+}$ and $\mathrm{Pb}^{2+}$, in which $6 \mathrm{~s}^{2}$ valence electrons are not involved in the formation of a chemical bond with neighboring ions, but form the so-called isolated (lone) pairs of electrons. The ions with unsaturated bonds are characterized by the high polarizability and strongly tend to an extraordinary coordination, in particular, to the formation of a polyhedron without an inversion center, when stereochemical ions shift to one of polyhedral anions, thereby forming a local dipole moment. Such a polarized state enhances the structural instability and can lead to the formation of a collective ferroelectric state $[1,2]$. In addition, the presence of dipoles causes strong distortions of both their own polyhedra and neighboring octahedra containing $3 \mathrm{~d}$ ions. This mechanism ensures a direct coupling between magnetic and charge degrees of freedom in these crystals. 
It is well known that the multiferroic properties are highly sensitive to the magnetic structure of a material. In particular, it was established for the $\mathrm{GdFe}_{3}\left(\mathrm{BO}_{3}\right)_{4}$ compound, the magnetoelectric properties of which were studied first among the $R \mathrm{Fe}_{3}\left(\mathrm{BO}_{3}\right)_{4}$ family compounds with a huntite structure, that the electric polarization only exists in a certain magnetically ordered phase [3]. In addition, in our previous studies on the $\mathrm{PbMBO}_{4}$ isomorphic crystals [4, 5], we observed a noticeable magnetodielectric effect in the antiferromagnetically ordered $\mathrm{PbFeBO}_{4}$ compound, whereas in $\mathrm{PbMnBO}_{4}$ with the ferromagnetic ground state this effect was almost absent.

Recently, Oh et al. [6] have shown that the $\mathrm{Ni}_{3} \mathrm{TeO}_{6}$ compound exhibits a colossal magnetoelectric response at the spin-flop transition. They proposed to use such a transition in hysteresis-free magnetoelectric switching by minor variations in an external electric/magnetic field. In addition, anomalies of the magnetoelectric response were observed at the magnetic spin-flop transitions in the $R \mathrm{MnO}_{3}$ rare-earth manganite crystals $[7,8]$. Another example is a family of Haldane spin-chain $R_{2} \mathrm{BaNiO}_{5}$ magnets with different rare-earth ions ( $R=\mathrm{Gd}$, Dy, Ho, Tb, and Er). The correlation between the Neel temperature and magnetodielectric response near the transition to the magnetoelectric phase was reported in [9], where the single-ion anisotropy of a rare-earth ion was shown to be responsible for the magnetodielectric effect in these compounds. The correlation between the magnetoanisotropic and magnetodielectric properties was found in the $\mathrm{Cu}_{3} \mathrm{~B}_{2} \mathrm{O}_{6}$ compound [10]. Magnetodielectric anomalies around the magnetic phase transitions, both spontaneous and magnetic field-induced, were observed in several magnets, including $\mathrm{Co}_{4} \mathrm{Nb}_{2} \mathrm{O}_{9}$ [11], $\mathrm{Mn}_{0.95} \mathrm{Co}_{0.05} \mathrm{WO}_{4}$ [12], and $\mathrm{SrNdFeO}_{4}$ [13]. Thus, the magnetodielectric properties of these materials can be used to study magnetic phase diagrams [14].

Petrakovskii et al. synthesized first orthorhombic $\mathrm{Pb}_{2} \mathrm{Fe}_{2} \mathrm{Ge}_{2} \mathrm{O}_{9}$ antiferromagnetic single crystals with a weak ferromagnetic moment [15]. This compound contains the stereochemical $\mathrm{Pb}^{2+}$ ion and therefore is interesting for studying the magnetodielectric properties. Furthermore, the magnetization measurements of this crystal showed that an external magnetic field applied along the $c$ axis induces the spin-flop transition, so it would be interesting to investigate the magnetodielectric response in the vicinity of this transition.

In this work, we report on the thorough investigations of the magnetic, thermodynamic, and magnetodielectric properties of the $\mathrm{Pb}_{2} \mathrm{Fe}_{2} \mathrm{Ge}_{2} \mathrm{O}_{9}$ single crystals. We demonstrate that the weak ferromagnetic moment originates from the combination of the single-ion anisotropy and Dzyaloshinskii-Moriya exchange interaction and quantitatively estimate their contributions. We refined the magnetic anisotropy of the crystal and established significant magnetodielectric anomalies near the spin-flop transition at certain mutual orientations of the electric and magnetic fields.

\section{Experimental}

The $\mathrm{Pb}_{2} \mathrm{Fe}_{2} \mathrm{Ge}_{2} \mathrm{O}_{9}$ single crystals were grown using a pseudo-flux technique modification of the spontaneous crystallization method. To avoid foreign contaminations that could come from a flux material, only classical solvents contained in the chemical composition of the synthesized crystal were used. Single crystals of two types were investigated: type-A single crystals were grown using the procedure described in [15] and type- $\mathrm{B}$ single crystals were also grown in the $\mathrm{PbO}-\mathrm{Ge}_{2} \mathrm{O}_{3}$ solvent, but with a higher eutectic point $\left(768^{\circ} \mathrm{C}\right)$. The ratio between the basic composition and the solvent was 1: 2 . The type-B single crystals appeared to be more voluminous and had an $\boldsymbol{a b}$-plane cross-section approximately twice as much as that of the type-A crystals and additional (100) and (010) faces perpendicular to the $\boldsymbol{a}$ and $\boldsymbol{b}$ axis, respectively. The temperature regime for growing the type-B crystals differed from the regime used for the type-A crystals by a higher temperature of 
expose $\left(1070^{\circ} \mathrm{C}\right)$; all the rest parameters of the two regimes were the same. The single crystals of both types were mechanically withdrawn from a crucible, without using nitric acid solutions. X-ray diffraction analysis showed that the crystals of both types have the identical crystal structure parameters consistent with the parameters reported in [15].

The magnetization of the $\mathrm{Pb}_{2} \mathrm{Fe}_{2} \mathrm{Ge}_{2} \mathrm{O}_{9}$ single crystals was measured at the Kirensky Institute of Physics (Krasnoyarsk) using a conventional vibrating sample magnetometer (VSM) with a superconducting solenoid operating in the temperature range of 4.2-300 K and magnetic fields up to $70 \mathrm{kOe}$ [16]. Angular dependences of the magnetization were obtained using a Quantum Design MPMS-3 Magnetic Property Measurement System with a horizontal rotation option. To measure specific heat in the temperature range of $2-300 \mathrm{~K}$ and magnetic fields up to $50 \mathrm{kOe}$, a Quantum Design PPMS-9 Physical Property Measurement System was used.

To study the magnetodielectric effect, two parallel faces perpendicular to the $\boldsymbol{a}$ or $\boldsymbol{c}$ axis were prepared. The faces were coated by EPO-TEK H20E electrically conductive epoxy-based glue. The samples prepared in this manner represented capacitors, which made it possible to apply an electric field parallel to the $\boldsymbol{a}$ or $\boldsymbol{c}$ axis. An experimental setup for studying the magnetodielectric effect was placed into a VSM cryostat [16]. The electric capacitance was measured as a function of temperature and magnetic field with an Agilent E4980A LCR meter using a four-wire circuit at a frequency of $100 \mathrm{kHz}$.

\section{Results and discussion}

The magnetic properties of the type- $\mathrm{A} \mathrm{Pb}_{2} \mathrm{Fe}_{2} \mathrm{Ge}_{2} \mathrm{O}_{9}$ single crystals were thoroughly investigated in [15]. Recently, we have reanalyzed the data obtained and assumed that, during the magnetization measurements, the $\boldsymbol{a}$ and $\boldsymbol{b}$ axes were confused ${ }^{1}$. We performed the magnetic measurements

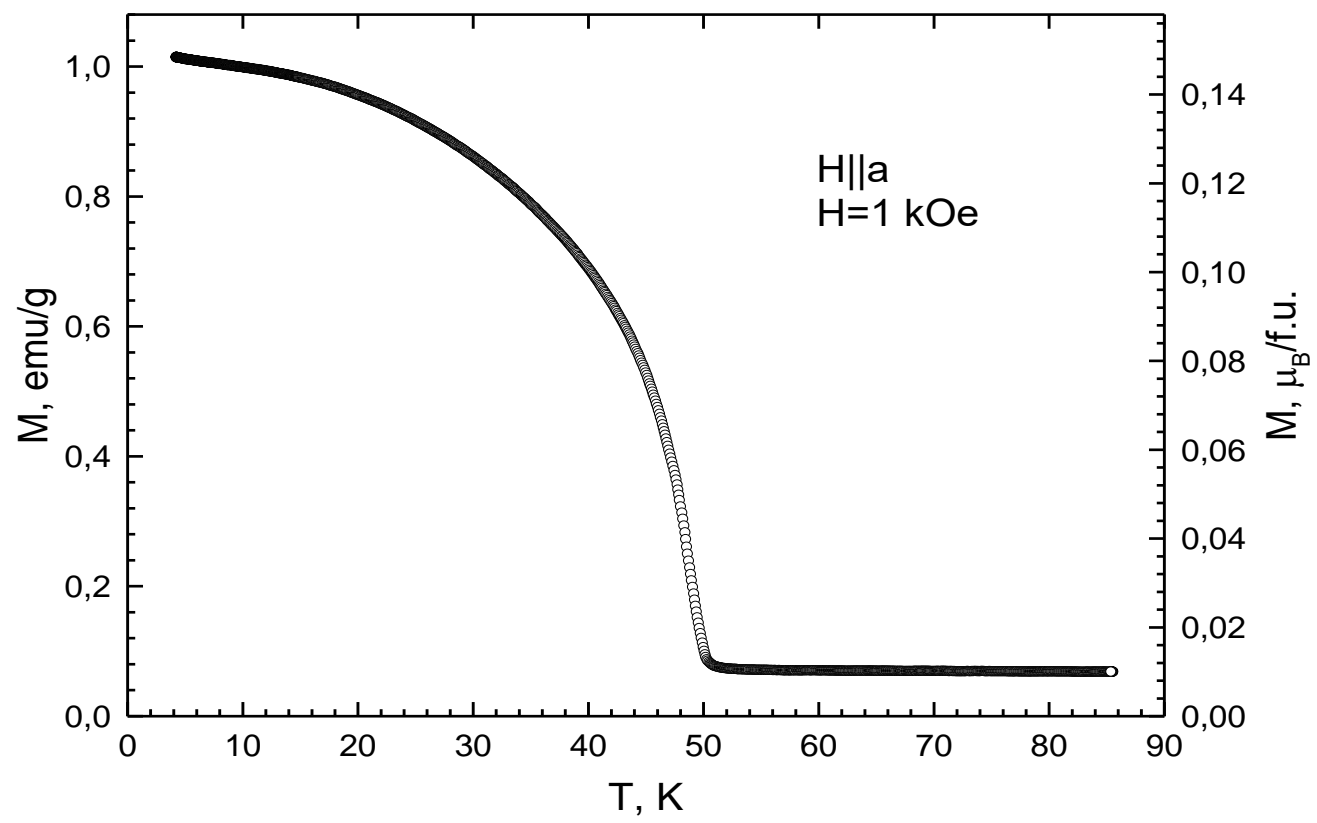

Fig. 1. Temperature dependence of the magnetization for the type- $\mathrm{B} \mathrm{Pb}_{2} \mathrm{Fe}_{2} \mathrm{Ge}_{2} \mathrm{O}_{9}$ crystal in a magnetic field of $\mathrm{H}=1 \mathrm{kOe}$ applied along the $\boldsymbol{a}$ axis.

${ }^{1}$ First, this was assumed by S.N. Martynov during the analysis of the symmetry properties of the crystal. 
on the carefully oriented type-B single crystals and confirmed our assumption. In particular, we found that, in contrast to the data from [15], the weak ferromagnetic moment is directed along the $\boldsymbol{a}$, rather than $\boldsymbol{b}$, axis. Figure 1 shows the temperature dependence of the magnetization measured along the $\boldsymbol{a}$ axis in a magnetic field of $1 \mathrm{kOe}$. This dependence is typical of the materials with a spontaneous magnetic moment. Both the low-temperature magnetization and Néel temperature values are consistent with the data reported in [15].

Figure 2 shows field dependences of the magnetization measured at $T=4.2 \mathrm{~K}$ along the three

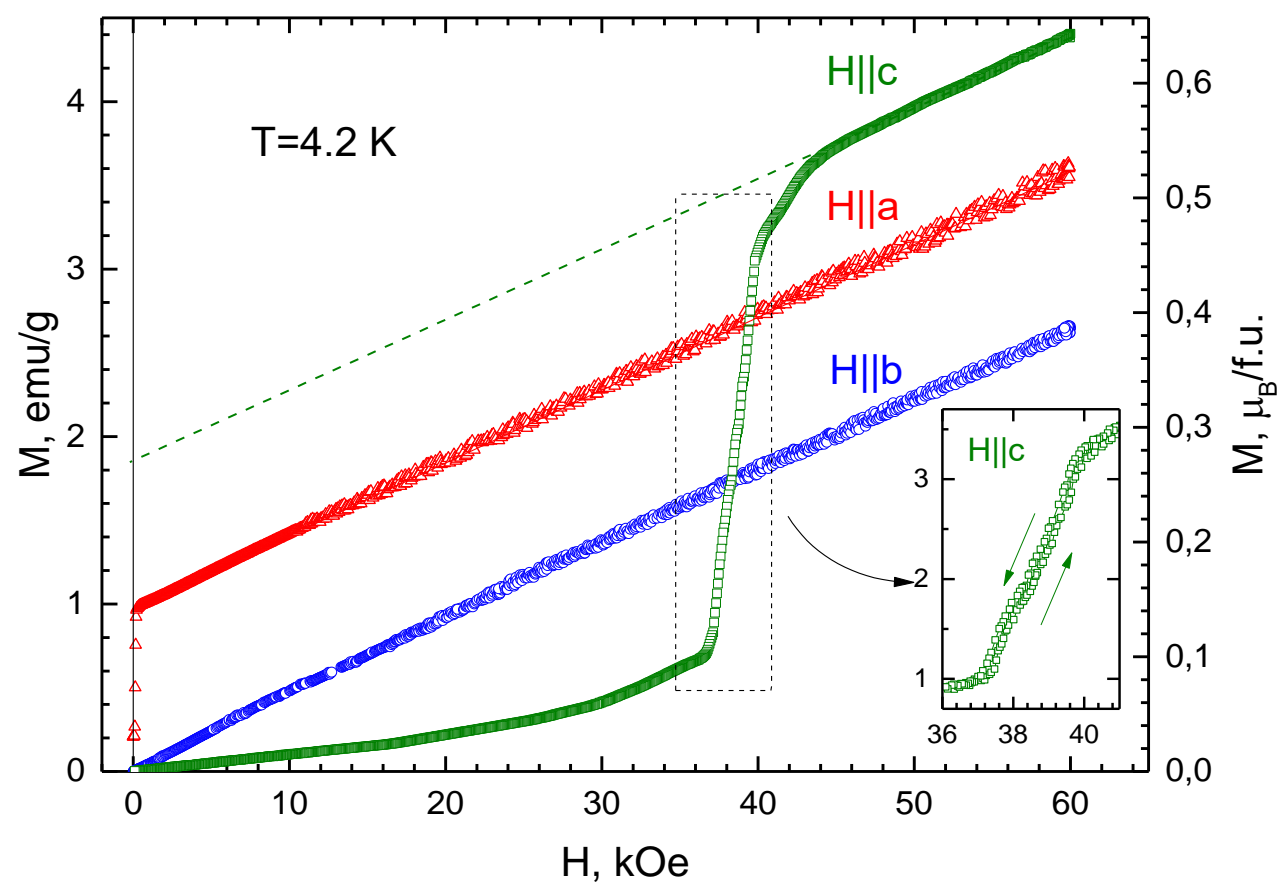

Fig. 2.Field dependences of the magnetization for the type- $\mathrm{B}_{2} \mathrm{Fe}_{2} \mathrm{Ge}_{2} \mathrm{O}_{9}$ single crystal measured at $T=4.2 \mathrm{~K}$ along the $\boldsymbol{a}, \boldsymbol{b}$ and $\boldsymbol{c}$ axes.

orthorhombic axes. They also confirm that the weak ferromagnetic moment is parallel to the [100] direction. The field dependence of the magnetization can be expressed as [17-19]

$$
\begin{aligned}
& M_{a}=M_{D a}+\chi_{\perp} H=\chi_{\perp}\left(H_{D a}+H\right) \\
& M_{D a}=\frac{H_{D a}}{H_{E}} M_{0}, \quad \chi_{\perp}=\frac{M_{0}}{H_{E}}
\end{aligned}
$$

Here, $M_{D a}=0.99 \mathrm{emu} / \mathrm{g}$ is the spontaneous weak ferromagnetic moment along the $\boldsymbol{a}$ axis, $\chi_{\perp}=$ $(4.33 \pm 0.005) \cdot 10^{-5} \mathrm{~cm}^{3} / \mathrm{g}, H_{D a}$ is the Dzyaloshinskii effective field, and $H_{E}$ is the exchange field. Using the experimental data obtained at $T=4.2 \mathrm{~K}$, we obtained a value of $H_{D a}=22.5 \mathrm{kOe}$, which is similar to a value of $21 \mathrm{kOe}$ from [15]; in the calculation, a value of $H_{E}=780 \mathrm{kOe}$ obtained from $\chi_{\perp}$ and a theoretical value of $M_{0}=230 \mathrm{G}$ were used.

In a magnetic field applied along the $\boldsymbol{b}$ axis, the magnetization increases linearly up to 60 kOe; however, we observed a sharp spin-flop transition in a magnetic field applied along the $c$ axis. The critical field $H_{s f}=39 \mathrm{kOe}$ of the spin-flop transition is noticeably weaker than a field of $\sim 47$ kOe reported for the type-A crystals in [15]. The weak hysteresis observed in the spin-flop vicinity indicates that this transition is first-order. 

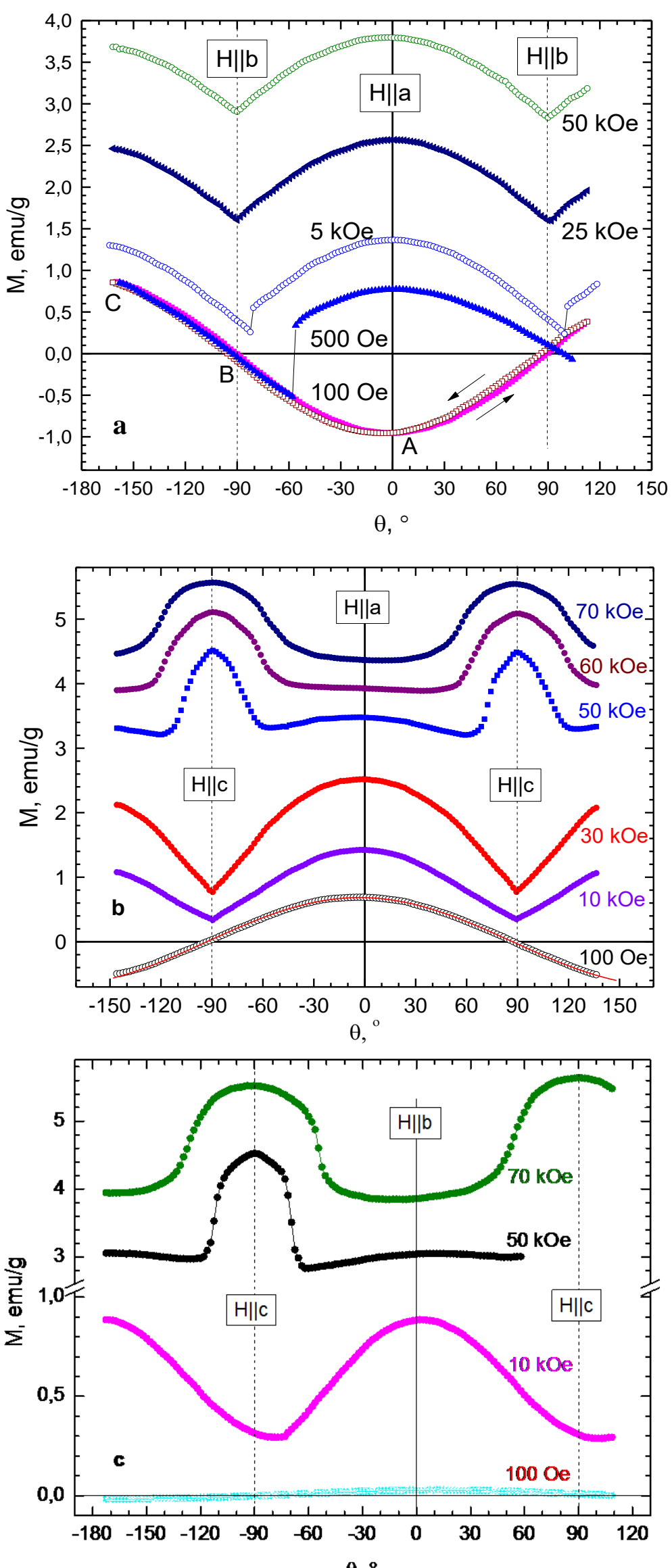

Fig. 3. Angular dependences of the magnetization measured at $T=2 \mathrm{~K}$ in the $\boldsymbol{a} \boldsymbol{b}$-plane (a), $\boldsymbol{a c}$-plane (b) and $\boldsymbol{b} \boldsymbol{c}$-plane (c) in various magnetic fields.

The critical field of the spin-flop transition is determined as a geometric mean of the effective 
anisotropy and exchange coupling fields [17]. Since the Néel temperature, which is mainly determined by the exchange coupling, is the same (or only slightly different) in the type-A and B crystals, the main contribution to the difference between the critical fields results from the effective field of the magnetic anisotropy. We may assumed that the type-A crystals exhibit the stronger magnetic anisotropy due to the additional contribution of the induced anisotropy, which could be guided by defects, strains, $\mathrm{Fe}^{2+}$ impurities, etc. occurring during the synthesis.

Above the spin-flop transition, the field dependence of the magnetization is described by an expression similar to (1), but with a different value of spontaneous weak ferromagnetic moment $M_{D c}$, which exceeds $M_{D a}$ by a factor of approximately 1.8. This value corresponds to a Dzyaloshinskii field of $H_{D c}=39.8 \mathrm{kOe}$ at $T=4.2 \mathrm{~K}$. As was shown in [19], the difference between the spontaneous moments measured along different orthorhombic axes is caused by the different ratios between the contributions of two mechanisms to the corresponding Dzyaloshinskii field, specifically, Dzyaloshinskii-Moriya and single-ion anisotropy:

$$
H_{D c}=D_{D M}+D_{S I} ; \quad H_{D a}=D_{D M}-D_{S I}
$$

Using Eq. (2), one can determine the corresponding contributions at $T=4.2 \mathrm{~K}: D_{D M}=31.2 \mathrm{kOe}$ and $D_{S I}=9.6 \mathrm{kOe}$. Note that the Dzyaloshinskii-Moria contribution dominates.

Angular dependences of the magnetization were measured on the type-A single crystal at $T=$ $2 \mathrm{~K}$ in the (100), (010), and (001) planes in different magnetic fields (Figs. 3a-3c). The angular dependences in the $\boldsymbol{a} \boldsymbol{b}$ plane (Fig. 3a) can be divided into three groups according to the external magnetic field value: weak fields $(H=100 \mathrm{Oe})$, medium fields $(H=500 \mathrm{Oe}$ and $H=5 \mathrm{kOe})$, and strong fields $(H>5 \mathrm{kOe})$.

In a weak magnetic field (100 Oe, Fig. 3a), the magnetization measured in the $\boldsymbol{a} \boldsymbol{b}$ plane takes both positive and negative values and obeys the simple cosine dependence $M \propto-\cos \theta$. To explain such a behavior, let us consider a simple qualitative model, taking into account that, in this plane, the magnetization with an arbitrary magnetic field orientation is a sum of components $M_{a}$ and $M_{b}$ and the dependence of $M_{a}$ on the field projection onto the $\boldsymbol{a}$ axis is determined by the hysteresis loop (see a schematic in Fig. 4). We assume that, in a weak field, the magnetization $M_{b}$ measured along the $\boldsymbol{b}$ axis and determined by the susceptibility $\chi_{\perp}$ is significantly weaker than the component $M_{a}$. Then, we may assume that, in weak fields, we have $M_{b}=0$ and the measured magnetization is only determined by the $M_{a}$ projection onto the field direction: $M \propto M_{a} \cdot \cos \theta$, where $\theta$ is the angle

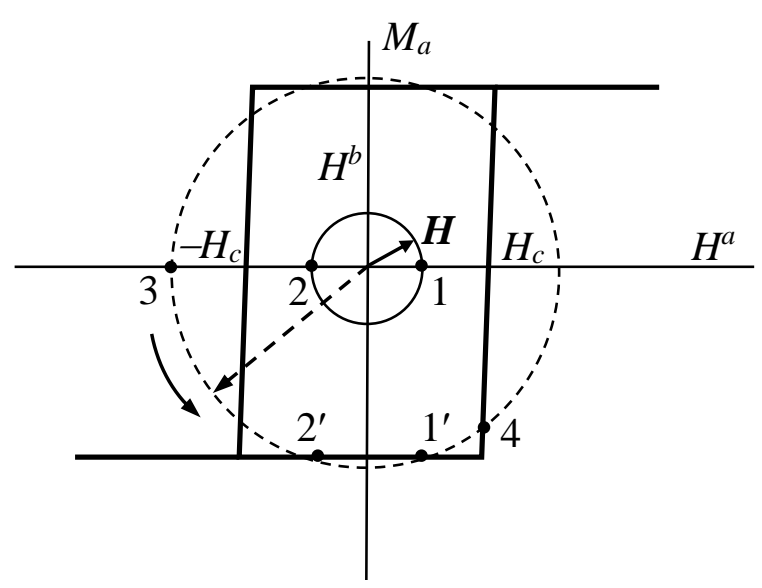

Fig. 4. Schematic of the field dependence of the magnetization measured along the $\boldsymbol{a}$ axis. between the field direction and $\boldsymbol{a}$ axis. In addition, we assume the external magnetic field $H=$ 100 Oe to be weaker than the coercivity $H_{c}$. Upon rotation of the field $H$ relative to the sample, as is shown schematically in the coordinates $\left(H^{a}\right.$, $H^{b}$ ) by a small circumference at the center of the loop, the field projection onto the $\boldsymbol{a}$ axis changes from +100 to -100 Oe between points 1 and 2 . If the initial state of magnetization $M_{a}$ corresponds to the negative branch of the loop, then $M_{a}$ changes between points $1^{\prime}$ and 2 , respectively, and remains in the negative branch of the hysteresis loop over the entire field rotation period. 
Therefore, when the field is parallel to the positive direction of the $\boldsymbol{a}$ axis corresponding to $\theta=0$, the external field and magnetization $M_{a}$ are antiparallel and the measured magnetization is $M=-M_{a}$. In the angular dependence shown in Fig. 3a, this value corresponds to point A. When the field is oriented along the $\boldsymbol{b}$ axis, the angle is $\theta=\pi / 2$ and the magnetization in this direction is $M=0$ (point $\mathrm{B}$ in the angular dependence). Finally, point $\mathrm{C}$ corresponds to the field orientation parallel to the magnetization $M_{a}: M=+M_{a}$. In addition, note that in the forward and reverse field rotation directions, there is a visible hysteresis in the angular dependence of the magnetization in a field of $100 \mathrm{Oe}$.

The angular dependence of the magnetization measured in a field of 500 Oe (Fig. 3a) between $-170^{\circ}$ and $-56^{\circ}$ is similar to that measured in a field of $100 \mathrm{Oe}$ and the magnetization is proportional to $-\cos \theta$. However, a sharp transition occurs at $\theta_{c}=-56^{\circ}$ and the angular dependence of the magnetization changes from $M \propto-\cos \theta t o M \propto+\cos \theta$. We explain this transition, assuming an external field of 500 Oe to be stronger than the coercivity $H_{c}$. A schematic of the field rotation trajectory is shown in Fig. 4 by a large dashed circumference. Let us assume that the initial state of magnetization $M_{a}$ corresponds to the negative branch of the hysteresis loop. Then, in the range of orientations from point 3 to point 4 along the arrow, the angular dependence of the magnetization coincides with the dependence measured in a field of 100 Oe. At point 4, the field projection onto the $\boldsymbol{a}$ axis becomes equal to the coercivity, $\left|H^{a}\right|=H_{c}$, and the magnetization $M_{a}$ abruptly jumps to the positive branch of the hysteresis loop. Upon further rotation of the sample, the angular dependence of the magnetization corresponds to $+\cos \theta$ unless it reaches the next critical point at $\theta_{c}=\theta_{c}+\pi$. We estimated the coercivity under the assumption $H \cdot \cos \theta_{c}=H_{c}$ and obtained a value of $H_{c}=290$ Oe. This estimated coercivity is similar to a value of 300-400 Oe from [15]. Similar jumps were also observed during the measurements in a field of $5 \mathrm{kOe}$, but in this field the magnetization $M_{b}$ becomes significant and its contribution leads to strong distortions of the hysteresis loop and a significant overestimation of the coercivity. In the stronger fields, the jumps between the upper and lower branches of the hysteresis loop occur directly when the magnetic field passes through the $\boldsymbol{b}$ axis direction and the projection of field $H^{a}$ changes its sign.

The angular dependences of the magnetization measured in the $\boldsymbol{a c}$ plane in different magnetic fields are shown in Fig. 3b. As in the previous case, when a magnetic field of 100 Oe is weaker than the coercivity, the contribution of component $M_{c}$ can be ignored and the angular dependence is described well by the function $M_{a} \cdot \cos \theta$ shown in the figure by a red solid line. In stronger fields (10 and $30 \mathrm{kOe})$, the angular dependences are described by the function $|\cos \theta|$ shifted along the $y$ axis by the value of magnetization $M_{c}$ along the $c$ axis, which is determined by the parallel magnetic susceptibility $\chi \|$.

The angular dependences measured in magnetic fields of 50-70 kOe above the critical spinflop transition field evoke special interest. In this case, when the field orientations are close to the $c$ axis, the angular dependences reveal a sharp increase in the magnetization due to the transition to the spin-flop state.

The similar angular dependences with anomalies caused by the spin-flop transition were obtained in a magnetic field rotated in the $\boldsymbol{b c}$ plane (Fig. 3c). During the measurements of the angular dependence in the $\boldsymbol{b} \boldsymbol{c}$ plane, a minor (approximately $2^{\circ}$ ) sample misorientation leads to the occurrence of a small contribution of the magnetization $M_{a}$, which can be observed during the measurements in relatively weak fields. As a result, a weak alternating angular dependence $\sim M \cos \theta$ was observed in a field of $100 \mathrm{Oe}$. In addition, the angular dependence obtained in a field of $10 \mathrm{kOe}$ contains a singularity at $\theta=-73^{\circ}$, which is characteristic of a jump of magnetization $M_{a}$ between the positive and negative branches of the hysteresis loop. 
We would like to emphasize that all the angular dependences of the magnetization unambiguously show that the principal magnetic anisotropy directions in the $\mathrm{Pb}_{2} \mathrm{Fe}_{2} \mathrm{Ge}_{2} \mathrm{O}_{9}$ single crystal coincide with the orthorhombic crystallographic axes. We observed the different behavior in the $\mathrm{Pb}_{2} \mathrm{Fe}_{2} \mathrm{Ge}_{2} \mathrm{O}_{9}$ crystal with partial substitution of manganese ions for iron; the results obtained will be published elsewhere.

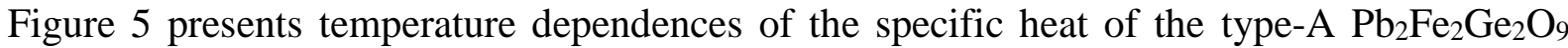
single crystal measured in fields of 0,10 , and $47 \mathrm{kOe}$. Near the magnetic transition, one can see a typical $\lambda$-like anomaly and the Néel temperature was found to be $T_{N}=(45.2 \pm 0.5) \mathrm{K}$. It was established that the specific heat is temperature-independent within the experimental accuracy.

At a temperature of about $30 \mathrm{~K}$, a specific heat anomaly is observed in a magnetic field of 47 kOe applied parallel to the orthorhombic $c$ axis, which was not found during the measurements in different fields and without field. In the right-hand inset in Fig. 5, this portion of the temperature dependence is shown in an enlarged scale. This anomaly is apparently caused by the transition to the spin-flop state, since the transition temperature coincides with the magnetic phase diagram data for this single crystal from [15].

To estimate the lattice contribution, the temperature dependence of the specific heat in zero magnetic field in the range of 60-300 K was fitted by a sum of the Debye and Einstein contributions. The calculated curve with parameters of $\theta_{D}=271 \mathrm{~K}$ and $\theta_{E}=775 \mathrm{~K}$ is shown by a red solid line on the main panel and in the left-hand inset in Fig. 5. Subtracting this contribution from the experimental dependence, we arrive at the temperature dependence of the magnetic contribution to

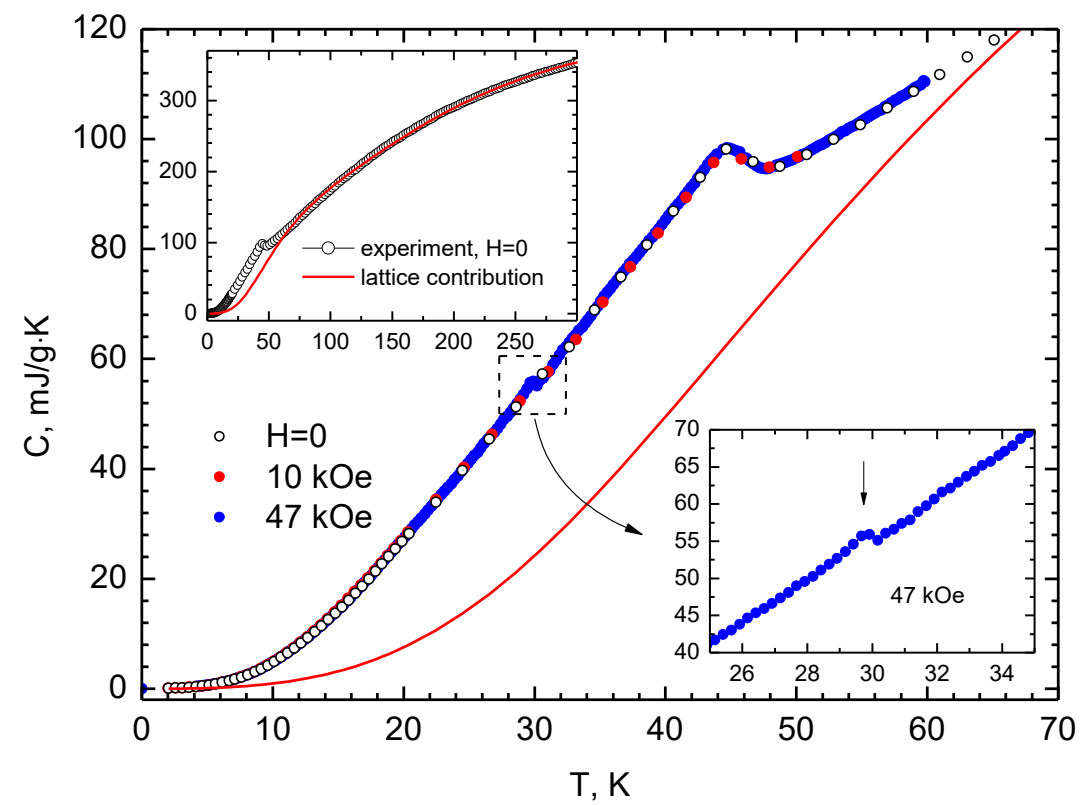

Fig. 5.Temperature dependences of the specific heat of $\mathrm{Pb}_{2} \mathrm{Fe}_{2} \mathrm{Ge}_{2} \mathrm{O}_{9}$ in different magnetic fields. Points show the experiment and a solid line, the lattice contribution. Left-hand inset: dependence of the specific heat in zero field in the extended temperature range. Right-hand inset: fragment of the dependence obtained in a magnetic field of $47 \mathrm{kOe}$.

the specific heat shown in Fig. 6a. Upon cooling, the magnetic contribution arises at $T \approx 70 \mathrm{~K}$ and sharply increases at approaching $T_{\mathrm{N}}$. 


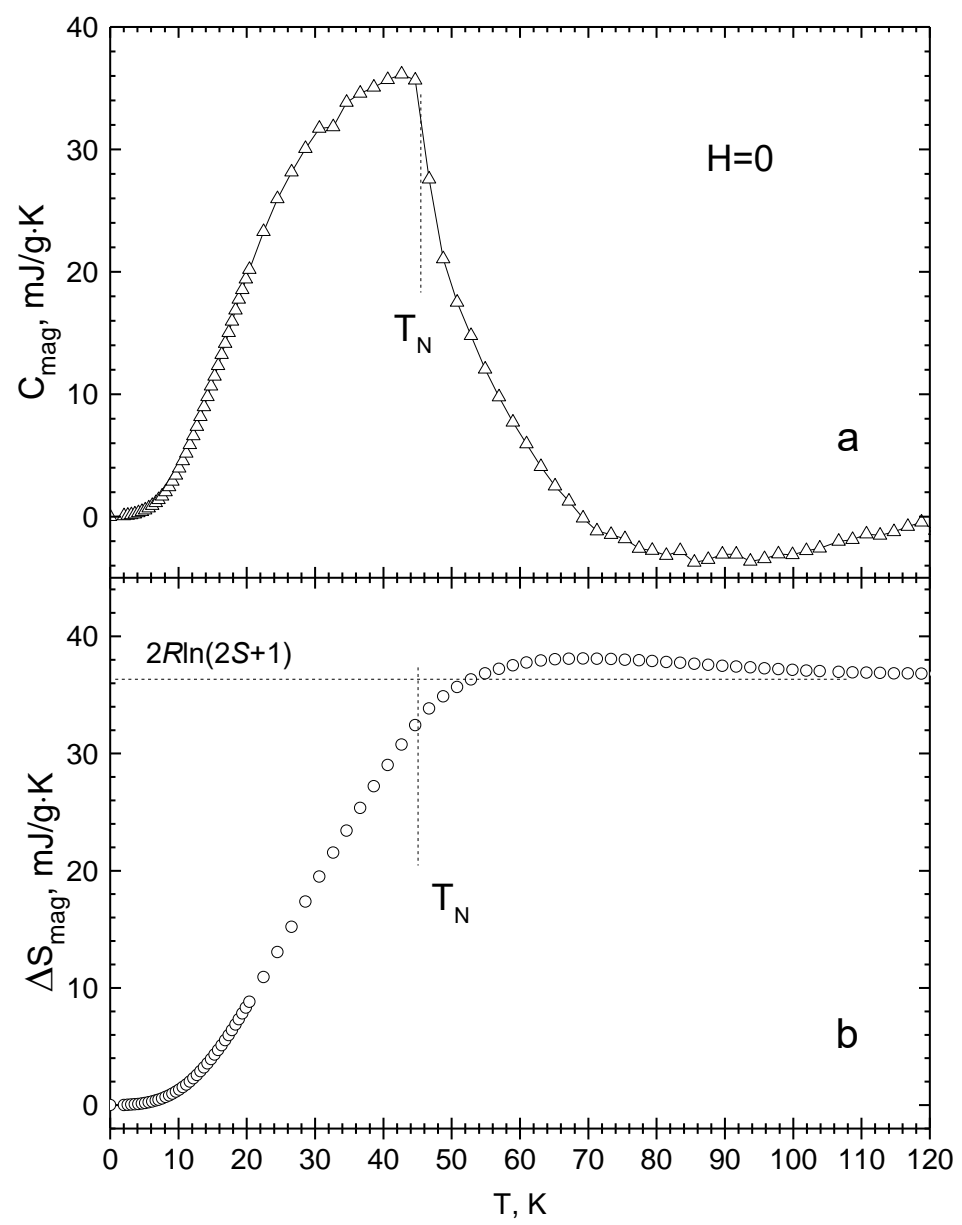

Fig. 6.Temperature dependences of the magnetic contributions to (a) the specific heat and (b) entropy for the $\mathrm{Pb}_{2} \mathrm{Fe}_{2} \mathrm{Ge}_{2} \mathrm{O}_{9}$ crystal.

Having estimated the magnetic contribution to the specific heat, we calculated the magnetic

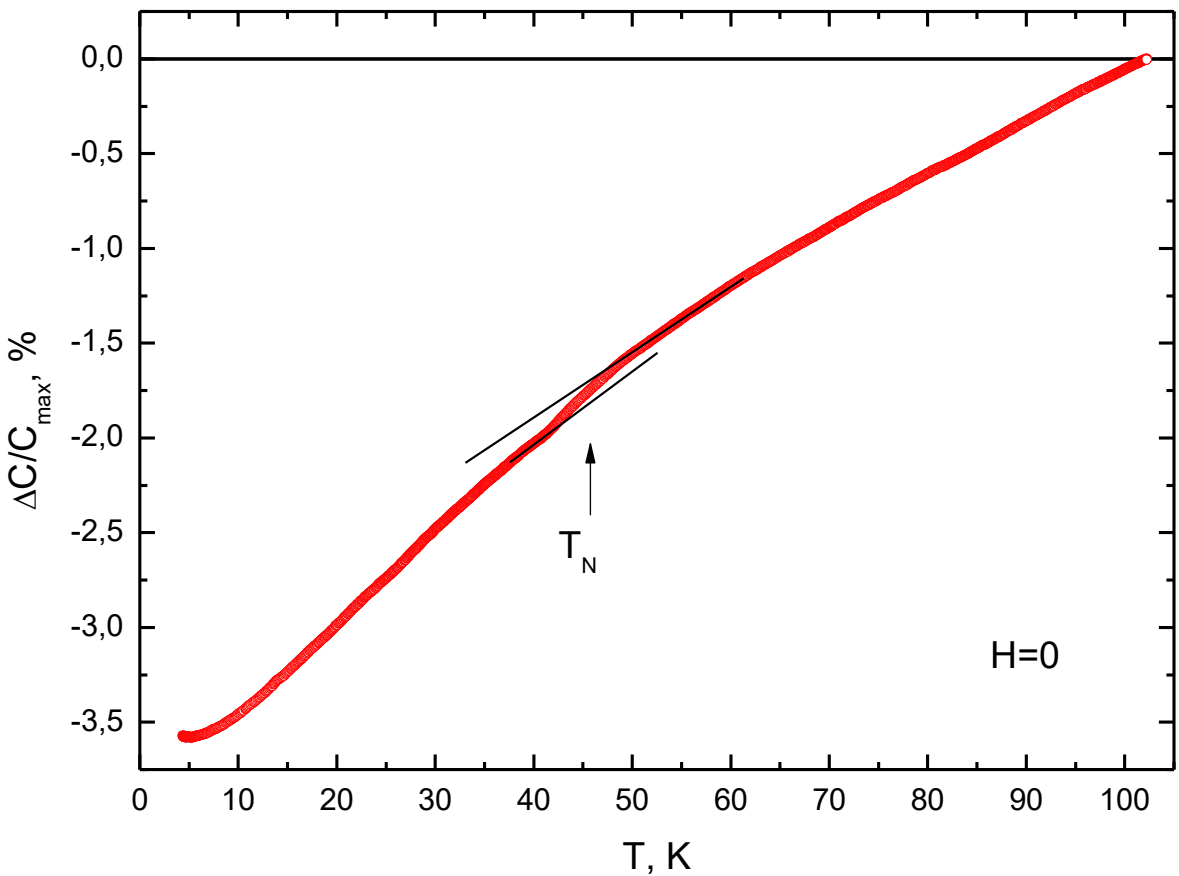

Fig. 7. Temperature dependence of the relative change in the capacitance of a capacitor with the $\mathrm{Pb}_{2} \mathrm{Fe}_{2} \mathrm{Ge}_{2} \mathrm{O}_{9}$ single crystal. The electric field is $\mathbf{E} \| \boldsymbol{c}$. 
entropy related to the phase transition. The temperature dependence is shown in Fig. 7b. Upon heating to the Néel temperature, the magnetic contribution to the entropy attains a value determined as $\Delta S_{\text {mag }}=2 R \ln (2 S+1)=36.54 \mathrm{~mJ} / \mathrm{g} \cdot \mathrm{K}$, which is indicative of a purely magnetic nature of the transition.

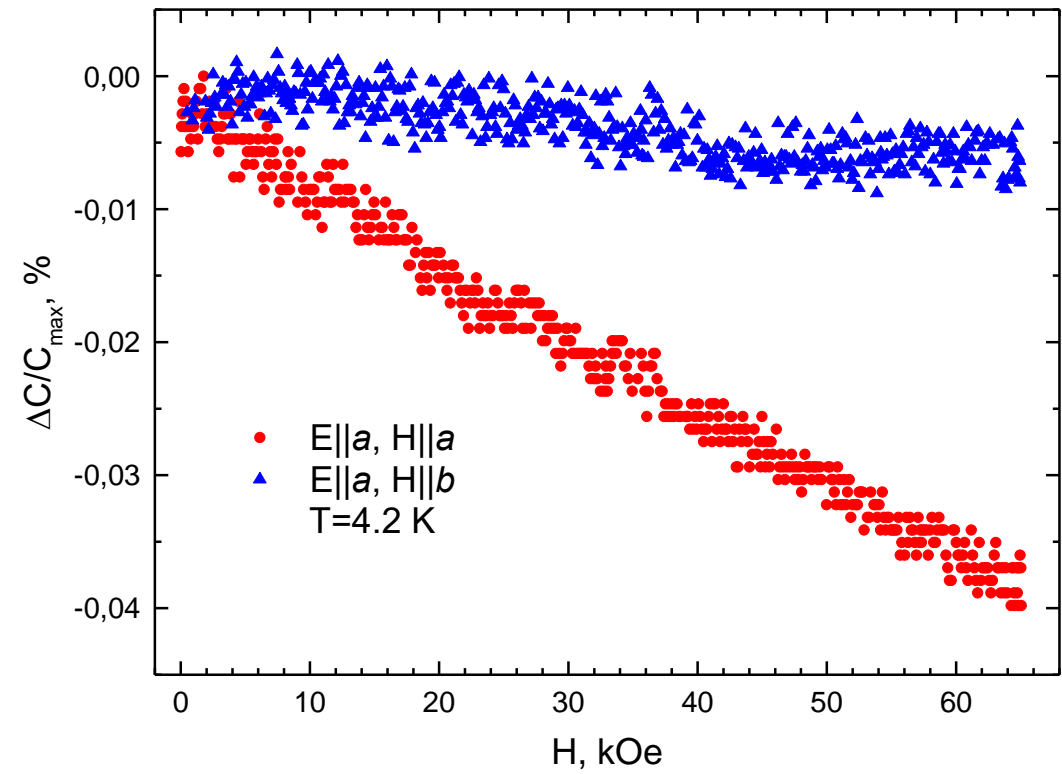

Fig. 8.Magnetic field dependences of the magnetodielectric response for the $\mathrm{Pb}_{2} \mathrm{Fe}_{2} \mathrm{Ge}_{2} \mathrm{O}_{9}$ single crystals. The magnetic field lies in the $\boldsymbol{a b}$ plane.

To study the magnetodielectric effect, two type-B samples in the form of plane-parallel plates were prepared so that the electric field could be applied along the $\boldsymbol{a}$ and $\boldsymbol{c}$ axes. It is worth noting that the initial single crystals were fairly small and we could not prepare samples in a form corresponding to a flat capacitor model. Therefore, the measurement data are given in relative units of the capacitance rather than permittivity.

Figure 7 shows the temperature dependence of the relative change in the capacitance of a capacitor with the $\mathrm{Pb}_{2} \mathrm{Fe}_{2} \mathrm{Ge}_{2} \mathrm{O}_{9}$ single crystal. The change in the capacitance was normalized to its maximum value. It can be seen that the temperature dependence of $\Delta C / C_{\max }$ changes its slope near the Néel temperature.

The field dependences of the magnetodielectric effect measured at $T=4.2 \mathrm{~K}$ and different mutual orientations of the electric and magnetic fields are shown in Figs. 8 and 9. Figure 8 presents the dependences obtained in a magnetic field applied in the $\boldsymbol{a b}$ plane. In these geometries, we found a small magnetodielectric response only when both the electric and magnetic fields are parallel to the $\boldsymbol{a}$ axis, whereas for all the other possible combinations of the magnetic and electric field direc-

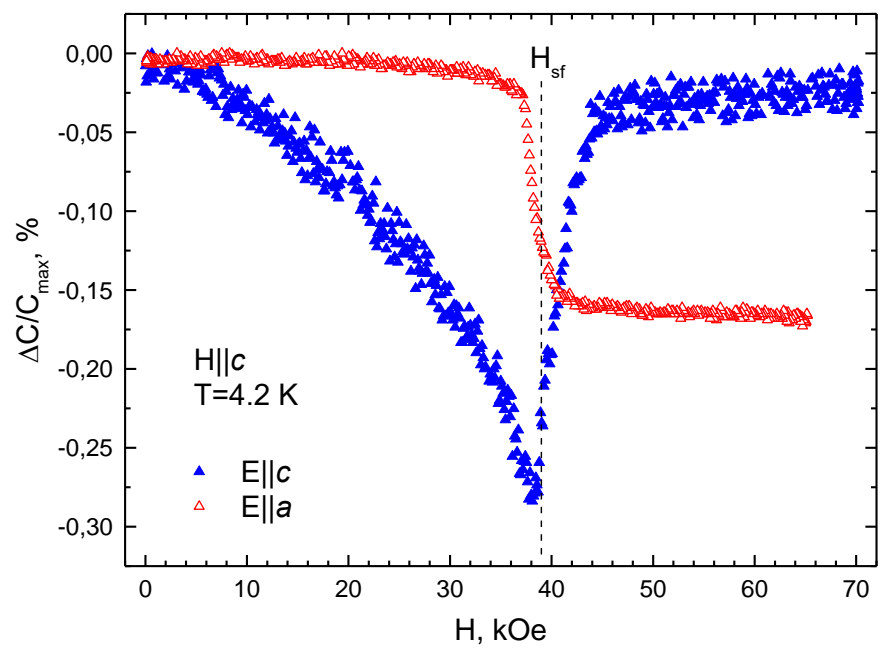

Fig. 9. Magnetic field dependences of the magnetodielectric response of the $\mathrm{Pb}_{2} \mathrm{Fe}_{2} \mathrm{Ge}_{2} \mathrm{O}_{9}$ single crystals. The magnetic field is $\mathbf{H} \| \boldsymbol{c}$ (the spin-flop critical field is shown by a dashed line). 
tions, the dielectric response was almost independent of the magnetic field.

The more pronounced magnetodielectric effect was observed in a magnetic field applied along the $c$ axis. In this case, we found the most prominent anomalies of the magnetodielectric response for both electric field orientations, $\mathbf{E} \| \boldsymbol{a}$ and $\mathbf{E} \| \boldsymbol{c}$ (Fig. 9). Taking into account that these anomalies are observed in the vicinity of the spin-flop transition, we attributed them to the magnetic structure transformation during the transition. The form of the anomaly depends on the electric field orientation: at $\mathbf{E} \| \boldsymbol{a}$, the capacitance is independent of the magnetic field in the range of $H<H_{s f}$, then, it changes abruptly at the transition and has a plateau above $H_{s f}$. At $\mathbf{E} \| \boldsymbol{c}$, the capacitance continuously decreases with increasing field at $H<H_{s f}$, abruptly returning to the initial level during the transition, and also remains almost constant above $H_{s f}$. Similar results were obtained for the type-A crystals.

\section{Discussion. Origin of the magnetodielectric response}

The results obtained in this work do not allow us to unambiguously establish the origin of the magnetodielectric response. In our experiments, we studied the capacitance $C \propto \varepsilon / d$ ( $d$ is the distance between the conductors and $\varepsilon$ is the permittivity of the material) of a flat capacitor-like device with $\mathrm{Pb}_{2} \mathrm{Fe}_{2} \mathrm{Ge}_{2} \mathrm{O}_{9}$ as a dielectric medium. Therefore, one of possible explanations for this effect is the magnetostriction field-induced change in the sample size. If we base on the strongest effect $\Delta \mathrm{C} / \mathrm{C}=$ $0.3 \%$ found in the experiment configuration $\mathbf{E}\|\boldsymbol{a}-\mathbf{H}\| \boldsymbol{c}$ and assume this capacitance change to be determined exclusively by an increase in the distance between the capacitor plates due to the magnetostriction with constant $\varepsilon$, we obtain the estimated magnetostriction constant $\lambda \sim 3 \cdot 10^{-3}$. However, such a magnetostriction value is quite doubtful for a compound with $\mathrm{Fe}^{3+}$ ions, which have no orbital moment. The magnetostriction constant in such materials is usually lower by two-three orders of magnitude. For example, in the $\mathrm{YFe}_{3}\left(\mathrm{BO}_{3}\right)_{4}$ antiferromagnet with a Néel temperature of $T_{N}$ $=38 \mathrm{~K}$ similar to that of $\mathrm{Pb}_{2} \mathrm{Fe}_{2} \mathrm{Ge}_{2} \mathrm{O}_{9}$, the magnetostriction is $\lambda=2 \cdot 10^{-6}$ at $T=4.2 \mathrm{~K}$ [20]. In the antiferromagnetic hematite $\alpha-\mathrm{Fe}_{2} \mathrm{O}_{3}$, the maximum magnetostriction constants are no higher than $\lambda$ $=1.4 \cdot 10^{-5}$ [21]. If we assume, in addition, that, due to the magnetostriction, the permittivity $\varepsilon$ changes as a result of a decrease in the dipole concentration, i. e., $\varepsilon \sim 1 / d$, then it requires a magnetostriction of $\lambda \sim 1.5 \cdot 10^{-3}$, which also exceeds by far all reasonable expectations. A similar assumption was made concerning the magnetostriction nature of the magnetodielectric effect in the $\mathrm{SrNdFeO}_{4}$ antiferromagnet with $\mathrm{Nd}$ ions characterized by a strong spin-orbit coupling [13]; however, even in such a compound, it was mentioned that too high magnetostriction is required to ensure the experimental ratio $\Delta C / C \sim 0.9 \cdot 10^{-3}$.

Another possible scenario suggests the variation in the permittivity $\varepsilon$ during the spin-flop transition. Adem et al. [22] showed that the strong magnetodielectric response of $\mathrm{TbFe}_{3}\left(\mathrm{BO}_{3}\right)_{4}$ in the vicinity of the metamagnetic transition cannot be explained by the magnetostriction effect and should be attributed to the coupling of magnetic excitations with transverse optical phonon modes and distortions of $\mathrm{FeO}_{6}$ octahedra above the metamagnetic transition. Another example of a material with the pronounced magnon-phonon coupling is $\mathrm{PbFeBO}_{4}$, where the anomalous temperature evolution of several phonon modes was observed near the $T_{\mathrm{N}}$ [23]. However, to accept one of these scenarios, it would be useful to measure the magnetostriction effect or high-resolution X-ray diffraction above the spin-flop state. Furthermore, inelastic neutron scattering or Raman-scattering spectroscopy data on the field dependence of optical phonons below and above spin-flop the transi- 
tion could give an insight to the extraordinary magnetodielectric properties of the $\mathrm{Pb}_{2} \mathrm{Fe}_{2} \mathrm{Ge}_{2} \mathrm{O}_{9}$ single crystals.

\section{Conclusions}

Using the modified pseudo-flux technique, the $\mathrm{Pb}_{2} \mathrm{Fe}_{2} \mathrm{Ge}_{2} \mathrm{O}_{9}$ orthorhombic antiferromagnet single crystals with a weak ferromagnetic moment were synthesized. The static magnetic properties of the crystals were thoroughly investigated and the dependence of the critical field of the spin-flop transition on the synthesis conditions was found. The weak ferromagnetic properties of the crystal were investigated for the magnetizations along both the orthorhombic $\boldsymbol{a}$ and $\boldsymbol{c}$ axis in the spin-flop state. It was concluded from the difference between the Dzyaloshinskii fields for these two directions that the weak ferromagnetism of this crystal results from the interplay between the single-ion anisotropy and antisymmetric Dzyaloshinskii-Moriya exchange coupling with the latter contribution being dominant.

An analysis of the angular dependences of magnetization showed that, in the magnetic fields weaker than the coercivity, the spontaneous moment is frozen in one of the hysteresis loop branches over the entire field rotation period. As a result, the angular dependence is alternating and described well by the function $\sim M_{a} \cos \theta$. In strong magnetic fields rotating in the $\boldsymbol{a c}$ and $\boldsymbol{b c}$ planes, spin-flop transition anomalies were observed in the angular dependences. All the angular dependences of the magnetization showed that the symmetries of the magnetic and crystal structures of $\mathrm{Pb}_{2} \mathrm{Fe}_{2} \mathrm{Ge}_{2} \mathrm{O}_{9}$ are identical.

The thermodynamic properties of the $\mathrm{Pb}_{2} \mathrm{Fe}_{2} \mathrm{Ge}_{2} \mathrm{O}_{9}$ crystals were studied and the Néel temperature $T_{N}=45.2 \mathrm{~K}$ independent of the magnetic field was determined from the temperature dependence of the heat capacity. The temperature dependences of the magnetic contributions to the specific heat and entropy were built. The magnetic entropy attains $2 R \ln (2 S+1)$ right above $T_{\mathrm{N}}$, which is indicative of a purely magnetic nature of the transition. In a magnetic field of $47 \mathrm{kOe}$ applied along the orthorhombic $c$ axis, an anomaly caused by the spin-flop transition was observed in the temperature dependence of the specific heat.

The magnetodielectric properties of the $\mathrm{Pb}_{2} \mathrm{Fe}_{2} \mathrm{Ge}_{2} \mathrm{O}_{9}$ compound were examined at different mutual orientations of the electric and magnetic fields and the most prominent anomalies were found at $\mathbf{H} \| \boldsymbol{c}$ in the vicinity of the spin-flop transition. The estimation showed that the attempts to explain the magnetodielectric effect by the magnetostrictive change in the sample geometry yield the magnetostriction constants two-three orders of magnitude larger than reasonable values. Another possible explanation of the effect can be based on the permittivity variation at the spin-flop transition due to the magnon-phonon coupling.

\section{Acknowledgments}

The authors thank S. N. Martynov for fruitful discussions.

This study was supported by the Russian Foundation for Basic Research, project no. 16-0200563; the reported study was also funded by RFBR and the Government of Krasnoyarsk Territory, Krasnoyarsk Regional Fund of Science, to the research project no. 18-42-240008 "Effect of magnetic structure on the magnetodielectric properties of oxide crystals containing stereoactive ions $\mathrm{Pb}^{2+}$ and $\mathrm{Bi}^{3+}$. S.E.N. acknowledges the support of the International Max Planck Research School for Chemistry and Physics of Quantum Materials (IMPRS-CPQM). 


\section{References}

[1] D.I. Khomskii, JMMM 306, 1 (2006).

[2] S. Ivanov, R. Tellgren, H. Rundlof, N. Thomas, and S. Ananta, J. Phys.: Cond. Matter 12 (2000) 2393.

[3] A.K.Zvezdin, S.S. Krotov, A.M. Kadomtseva, G.P. Vorob'ev, Yu.F. Popov, A.P. Pyatakov, L. N. Bezmaternykh, and E.N. Popova, JETP Letters 81 (2005)272.

[4] A. Pankrats, K. Sablina, D. Velikanov, A. Vorotynov, O. Bayukov, A. Eremin, M. Molokeev, S. Popkov, and A. Krasikov, JMMM 353 (2014) 23.

[5] A. Pankrats, K. Sablina, M. Eremin, A. Balaev, M. Kolkov, V. Tugarinov, and A. Bovina, JMMM 414 (2016) 82.

[6] Y.S. Oh, S. Artyukhin, J.J. Yang, V. Zapf, J.W. Kim, D. Vanderbilt, and S.-W. Cheong, Nature Communications 5 (2014) 3201.

[7] A.M. Kadomtseva, Yu.F. Popov, G.P. Vorob'ev, V. Yu. Ivanov, A.A. Mukhin, and A.M. Balbashov, JETP Letters 81 (2005) 590.

[8] A.M. Kadomtseva, Yu.F. Popov, G.P. Vorob'ev, K.I. Kamilov, A.P. Pyatakov, V. Yu. Ivanov, A.A. Mukhin, and A.M. Balbashov, JETP Letters 81 (2005) 19.

[9] S.K. Upadhyay, P.L. Paulose, and E.V. Sampathkumaran, Phys. Rev. B 96 (2017) 014418.

[10] D.A. Balaev, K.A. Sablina, A.L. Freydman, A.A. Krasikov, and A.F. Bovina, Phys. of the Solid State 58 (2016) 277.

[11] T. Kolodiazhnyi, H. Sakurai, and N. Vittayakorn, Appl. Phys. Lett. 99 (2011) 132906.

[12] I. Urcelay-Olabarria, E. Ressouche, Z. Wang, Yu. Skourski, V.Yu. Ivanov, Y.F. Popov, G.P. Vorobev, A.M. Balbashov, N. Qureshi, J. L. García-Muñoz, V. Skumryev, and A.A. Mukhin, Phys. Rev. B 96 (2017) 104435.

[13] J. Hwang, E.S. Choi, H.D. Zhou, Y. Xin, J. Lu,and P. Schlottmann, Phys. Rev. B 85 (2012) 224429.

[14] S.H. Bukhari, Th. Kain, M. Schiebl, A. Shuvaev, Anna Pimenov, A.M. Kuzmenko, X. Wang, S.-W. Cheong, J. Ahmad, and A. Pimenov, Phys. Rev. B 94 (2016) 174446.

[15] G.A. Petrakovskii, M.A. Popov, A.D. Balaev, K.A. Sablina, O.A. Bayukov, D.A. Velikanov, A.M. Vorotynov, A.F. Bovina, A.D. Vasil'ev, and M. Boehm, Phys. of the Solid State 51 (2009) 1745.

[16] A.D. Balaev, Yu.V. Boyarshinov, M.M. Karpenko, and B.P. Khrustalev, Prib. Tekh. Eksp. 3 (1985) 167.

[17] E.A. Turov, Physical Properties of Magnetically Ordered Crystals (Academic, New York, 1965).

[18] E.A. Turov, A.V. Kolchanov, V.V. Men'shenin, I.F. Mirsaev, and V.V. Nikolaev, Symmetry and Physical Properties of Antiferromagnets (Fizmatlit, Moscow, 2001) [in Russian].

[19] S.N.Martynov, JETP Letters 108 (2018) 196.

[20] A.M. Kadomtseva, Yu.F. Popov, G.P. Vorob'ev, A.P. Pyatakov, S.S. Krotov, K.I. Kamilov, V.Yu. Ivanov, A.A. Mukhin, A.K. Zvezdin, A.M. Kuz’menko, L.N. Bezmaternykh, I.A. Gudim, and V.L. Temerov, Low Temperature Physics 36 (2010) 511.

[21] R.Z. Levitin, A.S. Pachomov, and V.A. Shchurov, Phys. Lett. 27A (1968) 603.

[22] U. Adem, L. Wang, D. Fausti, W. Schottenhamel, P. H. M. van Loosdrecht, A. Vasiliev, L.N. Bezmaternykh, B. Büchner, C. Hess, and R. Klingeler, Phys. Rev. B 82 (2010) 064406. 
[23] M.A. Prosnikov, A.N. Smirnov, V.Yu. Davydov, K.A. Sablina, and R.V. Pisarev, J. Phys.: Condens. Matter. 29 (2017) 025808. 